\title{
Intracranial Pressure for the Characterization of Different Types of Hydrocephalus: A Permutation Entropy Study
}

\author{
Tricia Adjei, Daniel Abásolo, Member, IEEE, and David Santamarta
}

\begin{abstract}
Hydrocephalus is a condition characterized by altered cerebrospinal fluid (CSF) dynamics and chronic rises in intracranial pressure (ICP). However, the reason why hydrocephalic physiologies fail to inhibit dangerously high ICP levels is not known. Infusion studies are used to raise ICP and evaluate CSF circulation disorders. In this pilot study, ICP signals recorded during infusion tests from 33 patients with normal pressure hydrocephalus and 36 patients having developed a secondary form of normal pressure hydrocephalus were characterized using Permutation Entropy (PE), a symbolic non-linear method to quantify complexity. Each ICP signal was divided into four epochs - baseline (before infusion begins), infusion, plateau, and recovery (after infusion has stopped) and the mean PE was calculated for each epoch. Statistically significant differences were found between PE for most epochs ( $\mathbf{p}<0.00833$, Bonferroni-corrected Wilcoxon tests), with a significant decrease in the plateau phase. However, differences between PE for normal pressure and secondary hydrocephalus were not significant. Results suggest that the increase in ICP during infusion studies is associated with a significant decrease in PE. PE analysis of ICP signals could be useful for increasing our understanding of CSF dynamics in normal pressure hydrocephalus.
\end{abstract}

\section{INTRODUCTION}

Hydrocephalus is a condition which is characterized by accumulations of cerebrospinal fluid (CSF), which then leads to pressure being exerted on the brain [1]. It can appear as a primary condition or as a secondary effect to stroke or head injury, among other possible causes [2]. A standard way of managing hydrocephalus consists in implanting a CSF shunt after surgery [3], although this procedure is not always successful, with patients not responding well to treatment [2].

In 1970 Katzman and Hussey [4] introduced a manometric test to enable the diagnosis and assessment of hydrocephalus in patients. The test involves infusing saline into the subarachnoid space whilst performing a lumbar puncture [4]. The test is comprised of using an infusion

*Research supported by the University of Surrey Faculty of Engineering and Physical Sciences Research Fund FRSF 14-24.

T. Adjei was with the Centre for Biomedical Engineering, Department of Mechanical Engineering Sciences, Faculty of Engineering and Physical Sciences, University of Surrey, Guildford, United Kingdom, and is now with Oxehealth, Oxford, United Kingdom.

D. Abásolo is with the Centre for Biomedical Engineering, Department of Mechanical Engineering Sciences, Faculty of Engineering and Physical Sciences, University of Surrey, Guildford, United Kingdom (phone: +44(0)1483682971; e-mail: d.abasolo@ surrey.ac.uk).

D. Santamarta is with the Servicio de Neurocirugía, Hospital Universitario, León, Spain. pump connected to a needle to infuse saline until the pressure of CSF reaches a steady state; this is detected using a manometer connected to the needle via a stopcock [4], [5]. At this point, an inadequate resorption of CSF can be validated, leading to a correct diagnosis of hydrocephalus [4]. Infusion tests can be split into four phases; initially, baseline intracranial pressure (ICP) is recorded for up to ten minutes before the infusion itself commences [5]. Once a plateau pressure is obtained, ICP is then allowed to steadily return to baseline levels [5]. In this pilot study, the different phases will be referred to as the baseline (before infusion), infusion, plateau, and recovery (after infusion has stopped).

The unknowns surrounding hydrocephalus and ICP are related to the physiology behind ICP rise, and in particular, the causes of hydrocephalus. Coupling these with the fact that hydrocephalus diagnosis has not changed significantly since the introduction of lumbar infusion tests [4], the analysis of CSF dynamics and ICP can shed light on this condition. ICP characterization in hydrocephalus with signal processing techniques could offer benefits in terms of understanding the condition's pathophysiology and improving diagnoses. In fact, there is recent evidence that non-linear analysis of ICP signals in adults with hydrocephalus does reveal a loss of complexity during infusion studies [6].

Permutation Entropy (PE), a symbolic non-linear technique, was introduced given that the vast majority of entropic, dimensional and exponential measures of complexity were not applicable to real-world noisy series [7]. PE calculates the complexity of a signal by identifying different patterns appearing in it [7]. Therefore, this method could be useful for ICP characterization, particularly the changes associated with lumbar infusion tests.

The hypothesis for this study is that the analysis of ICP signals from patients with hydrocephalus as a primary condition or as a secondary effect with PE will reveal characteristics that could be associated to the nature of the condition.

\section{MATERIALS AND METHODS}

\section{A. Intracranial Pressure Signals}

ICP signals of patients showing clinical symptoms of normal pressure hydrocephalus (progressive gait disturbance, memory problems and urinary incontinence) together with ventriculomegaly (Evans index $>0.30$ ) were recorded using a variant of the Katzman and Hussey test at the Hospital Universitario of León (Spain). After 5 minutes of baseline 
recording, infusion of a Ringer solution started at a rate of $1.5 \mathrm{ml} / \mathrm{minute}$ until a plateau was reached in the ICP signal. After the infusion stopped, CSF pressure was recorded until it decreased towards baseline levels. ICP recordings were acquired with a sampling frequency of $100 \mathrm{~Hz}$ and patients or a close relative gave their informed consent to participate in the study, which was approved by the local ethics committee.

69 signals were retrospectively selected for this study, with 33 patients with normal pressure hydrocephalus referred to here as primary - (age $=76.9 \pm 6.8$ years old, mean \pm standard deviation; 20 males and 13 females) and 36 patients having developed a secondary form of normal pressure hydrocephalus (age $=63.5 \pm 16.6$ years old, mean \pm standard deviation; 16 males and 20 females). For each recording, a neurosurgeon identified the epochs that were representative of the phases of the infusion test (baseline, early infusion, plateau, and recovery after infusion).

A finite impulse response Hamming window filter of order 41424 and band-pass range of $0.005-10 \mathrm{~Hz}$ was used to filter each signal, therefore retaining the slow-wave components with range of $0.0055-0.5 \mathrm{~Hz}$ [8]. Fig. 1 shows a representative ICP signal, before and after filtering.

\section{B. Permutation Entropy}

$\mathrm{PE}$ is based on the premise of measuring the entropy within a time series based on the probability of occurrence of all possible permutations within the embedded series [7]. Given a time series with samples $x(i), i=1,2, \ldots, N$, PE can be calculated as follows [7]:

1. Choose an embedding dimension $n$ and create an embedding vectors $n$ samples long, with a time delay $\tau$ between each data point selected to join the embedding vector $(x(i), x(i+\tau), \ldots, x(i+(n-1) \tau)$.

2. Assign the lowest data point in the embedding vector the first position, the second lowest data point the second position until all data points in the embedding vector have been replaced with their ranking order.

3. Repeat 1 and 2 until all possible embedding vectors have been created and ranked. PE is calculated as:

$$
P E(n, \tau)=-\sum_{v=1}^{k} P_{v} \ln P_{v},
$$

where $k$ is the number of different sub-sequence ranked vectors from the original time series and $P_{v}$ is the fraction of the sub-sequence ranked vectors.

4. PE can be then normalized [9] with (2):

$$
P E_{\text {normalized }}(n, \tau)=\frac{P E(n, \tau)}{\ln (n !)},
$$

Complex signals with a highly irregular and unpredictable nature will be characterized by a greater range of different embedding vectors and, therefore, a higher PE. A normalized $\mathrm{PE}$ value of 0 corresponds to a totally periodic signal [9].

The computation of PE relies on the selection of specific parameters, namely the embedding dimension and time delay. In this pilot study, the choice of parameters was largely driven by the desire to characterize the ICP signals on an epoch-wise basis. This meant that the entropies had to be computed in sliding windows; a $10 \mathrm{~s}$ long window was chosen to move along the ICP signals from beginning to end using a $1 \mathrm{~s}$ slide.

The window length $N$ has to be compatible with the embedding dimension so as to meet the recommendation that $N \geq n$ ! [7]. Embedding dimensions of 3 or 4 are too small to accurately track the dynamical changes in a signal [10] whilst as the dimension increases, PE's ability to discriminate between different groups decreases [9]. Hence, prior to the analyses of the signals, the value of $n$ ! was calculated for dimensions 5, 6 and 7 and it was found that a value of $n$ of 5 and a window length $N$ of $10 \mathrm{~s}$ was an appropriate compromise between computing time and number of embedding vectors contained in each window to capture the dynamics of the signal. The time delay $\tau$ was equal to 1 sample. Mean PE values were then found for each of the patient's baseline, infusion, plateau, and recovery epochs.

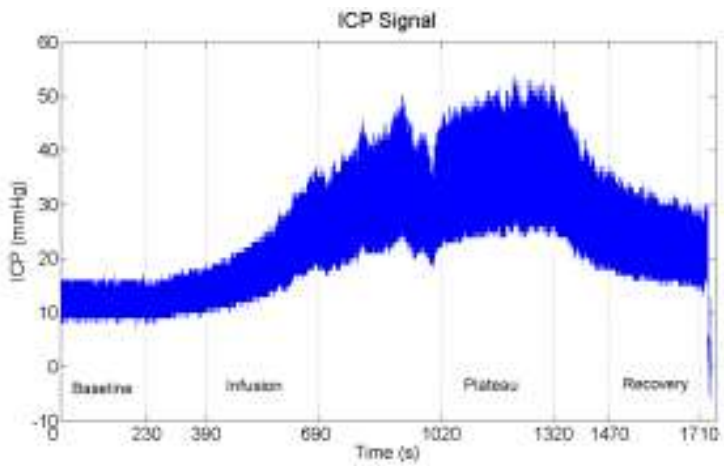

(a)

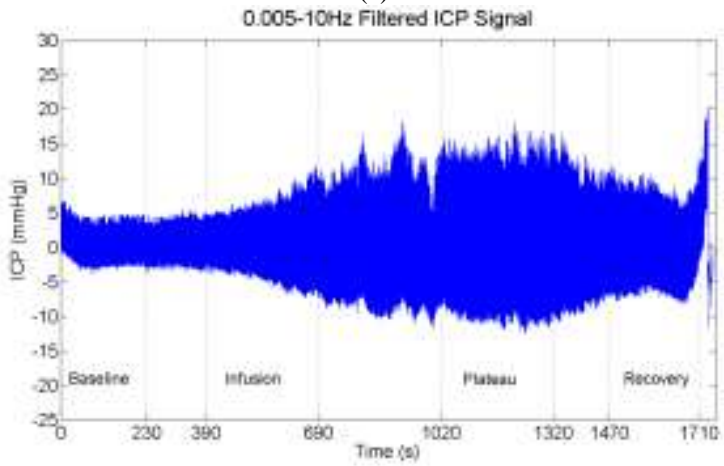

(b)

Fig. 1. Time domain plots of a representative ICP signal from a patient with normal pressure hydrocephalus. (a) Raw signal; (b) Filtered signal with a band-pass range of $0.005-10 \mathrm{~Hz}$. The four different epochs of the infusion test have been highlighted using vertical dashed lines. 


\section{Statistical Analysis}

The significance of the differences between the mean PE values for the different epochs in each group was assessed using the non-parametric Wilcoxon signed-rank test. The test was computed using a Bonferroni adjusted significance level of 0.00833 , which equates to 0.05 divided by 6 ; this accounts for the fact that though an overall significance level of 0.05 is desired, there would be a total of 6 comparisons between the four infusion test epochs.

To assess the significance of the differences between PE values for both groups, normality of the results was first checked using Lilliefors test. Student's t-test was then used to compare results from the groups with normal distributions, while the Kruskal-Wallis test was used when results did not follow a normal distribution. A significance level of 0.05 was used.

Lastly, receiver operating characteristic (ROC) curves were computed to offer an indication of how proficient PE is in discriminating between patients with primary and secondary forms of hydrocephalus, by assessing the diagnostic accuracy of the entropies [11]. ROC curves use decision rules with thresholds to determine the probability of correctly classifying a patient given a measured or derived quantity [11], comparing the rate of true negative diagnoses (in this case, the proportion of secondary cases correctly identified), termed specificity, with the rate of true positive diagnoses (percentage of primary cases correctly classified), termed sensitivity [12]. Each point on a ROC curve corresponds to the sensitivity obtained when using a particular threshold to separate patient groups, plotted against the corresponding 1-specificity value [12].

\section{RESULTS}

It was observed for both groups that PE decreased during the infusion test, reaching a minimum value during the plateau phase, and increasing again once infusion has stopped. Fig. 2 shows the normalized PE from the ICP signal plotted in Fig. (1), highlighting the changes in entropy observed in most signals. Table I summarizes the average PE results. Although it can be seen that PE was lower in both the plateau and recovery phases in the signals from patients with probable/possible normal pressure hydrocephalus than in those from patients having developed a secondary form of normal pressure hydrocephalus, differences were not significant $(\mathrm{p}>0.05$, Kruskal-Wallis test for baseline, Student's t-test for the other phases of the infusion test).

Table II and Table III summarize the results from the Wilcoxon signed-rank test for patients with normal pressure hydrocephalus and those with a secondary form of the condition, respectively. Statistically significant differences were found between all pairs, with the exception of the comparison between the early infusion and recovery epochs.

Table IV summarizes the ROC curve results, highlighting a low accuracy when discriminating between patients with primary and secondary forms of hydrocephalus.

\section{DISCUSSION}

In this pilot study, PE was used to characterize ICP signals. PE is a symbolic non-linear method faster than approximate or sample entropy, robust to noise, and invariant with respect to non-linear monotonous transformations [7]. Results show a significant decrease of $\mathrm{PE}$ values, associated with an increased regularity of the ICP signals, during infusion tests, reaching a minimum during the phase of the test where the pressure reached a plateau. These PE changes can be observed in both patients with normal pressure hydrocephalus and patients who have developed a secondary form of this condition, although for the latter the

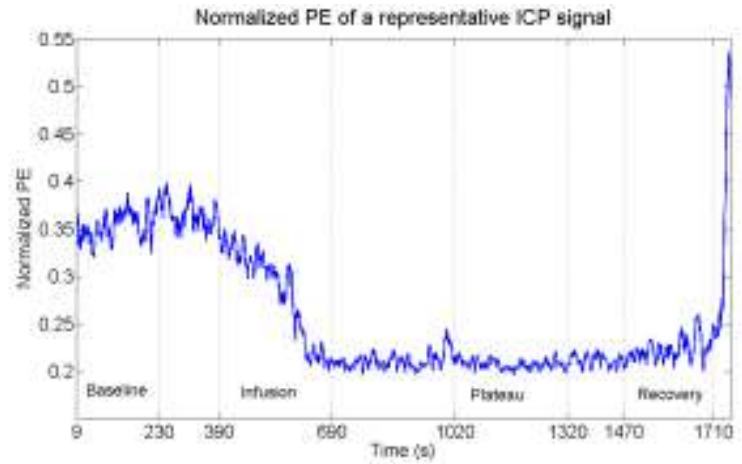

Fig. 2. Normalized PE of an ICP signal from a patient with normal pressure hydrocephalus. The four different epochs of the infusion test have been highlighted using vertical dashed lines.

TABLE I. MEAN NoRMALIZEd PE VALUES For Both GRouPs AND THE DiFFERENT EPOCHS OF THE INFUSION TESTS. RESULTS ARE PRESENTED AS MEAN \pm STANDARD DEVIATION

\begin{tabular}{|l|c|c|c|}
\hline & Primary & Secondary & p-value \\
\hline Baseline & $0.46 \pm 0.03$ & $0.47 \pm 0.03$ & $0.48^{\mathrm{a}}$ \\
\hline Infusion & $0.39 \pm 0.06$ & $0.39 \pm 0.08$ & $0.89^{\mathrm{b}}$ \\
\hline Plateau & $0.32 \pm 0.07$ & $0.34 \pm 0.10$ & $0.29^{\mathrm{b}}$ \\
\hline Recovery & $0.37 \pm 0.07$ & $0.39 \pm 0.07$ & $0.38^{\mathrm{b}}$ \\
\hline
\end{tabular}

a. Kruskal-Wallis test

b. Student's t-test

TABLE II. RESUlts OF THE COMPARISON BETWEEN PE VALUES For DIFFERENT EPOCHS IN PATIENTS WITH PRIMARY HYDROCEPHALUS

\begin{tabular}{|l|c|}
\hline & p-value \\
\hline Baseline vs. Infusion & $4.65 \cdot 10^{-10}$ \\
\hline Baseline vs. Plateau & $2.32 \cdot 10^{-10}$ \\
\hline Baseline vs. Recovery & $3.25 \cdot 10^{-9}$ \\
\hline Infusion vs. Plateau & $1.00 \cdot 10^{-8}$ \\
\hline Infusion vs. Recovery & 0.1115 \\
\hline Plateau vs. Recovery & $1.00 \cdot 10^{-8}$ \\
\hline
\end{tabular}

TABLE III. Results Of The Comparison Between PE VALues For DIFFERENT EPOCHS IN PATIENTS WITH SECONDARY HYDROCEPHALUS

\begin{tabular}{|l|c|}
\hline & p-value \\
\hline Baseline vs. Infusion & $1.24 \cdot 10^{-8}$ \\
\hline Baseline vs. Plateau & $4.07 \cdot 10^{-10}$ \\
\hline Baseline vs. Recovery & $4.07 \cdot 10^{-10}$ \\
\hline Infusion vs. Plateau & $2.50 \cdot 10^{-9}$ \\
\hline Infusion vs. Recovery & 0.7770 \\
\hline Plateau vs. Recovery & $2.05 \cdot 10^{-6}$ \\
\hline
\end{tabular}


reduction in PE values was slightly less than for the former.

Differences between PE values for primary and secondary forms of hydrocephalus were not statistically significant and the automatic classification of patients using ROC curves yielded low accuracies $(<60 \%)$. It is worth noting that it was possible to correctly classify $84.85 \%$ of patients with normal pressure hydrocephalus using PE values for the infusion phase, but at the expense of a very low classification rate for patients with a secondary form of the condition.

The physiological reason behind the reduction in entropy during pressure increases remains an enigma [7], [13]. Reductions in ICP complexity during hypertension have been attributed to the failure of cerebral auto-regulation [14]. It can be speculated that the similarly high basal PEs obtained for all signals could be indicative of healthy cerebral autoregulation prior to hypertension. Conversely, following the onset of infusion, the inference from [14] would suggest that cerebral auto-regulation degrades more substantially in normal pressure hydrocephalus than in secondary forms, as PE was lower during the plateau and recovery phases.

Some limitations of this study should be mentioned. The sample size was relatively small. As a result, our findings are preliminary and require replication in a larger database. Furthermore, information is lost about the magnitude of the patterns with PE [15]. As a result, some alternative versions of the original algorithm have been proposed to improve results. Modified PE takes into account the possible occurrence of equal values, by mapping the equal value onto the same symbol (rank) [9]. Weighted permutation entropy (WPE) is based on weighting permutation patterns depending on the amplitudes of their constituent data points [16]. It has been suggested that WPE overcomes the drawbacks of PE in the sense that vectors containing data points with large amplitude differences are not attributed with the same contribution to the final entropy as those with only small fluctuations caused by noise [16]. PE is also more susceptible to broad band noise than other alternatives [17]. Future research would address these issues and the influence of the values of $n$ and $\tau$ in PE. Moreover, results would be compared with those obtained with other algorithms, such as approximate, sample and multiscale entropies. Possible correlations with clinical parameters would be explored too.

To sum up, to the best of our knowledge this is the first study attempting the characterization of different forms of hydrocephalus from ICP signals. Differences between the entropies of signals from both groups provide support for the continued analysis of ICP signals based on the type of hydrocephalus. However, further research with a bigger database and other signal processing methods is needed.

\section{REFERENCES}

[1] E. N. Marieb and K. Hoehn, Human Physiology and Anatomy. $8^{\text {th }}$ ed. San Francisco: Pearson Benjamin Cummings, 2010.

[2] M. Bergsneider, C. Miller, P. M. Vespa and X. Hu, "Surgical management of adult hydrocephalus," Neurosurgery, vol. 62(Suppl 2), pp. 643-660, 2008.
TABLE IV. Classification Results ObTaINEd Using ROC CURVES TO CHECK THE PROFICIENCY OF PE IN DISCRIMINATING BETWEEN PATIENTS WITH PRIMARY AND SECONDARY FORMS OF HYDROCEPHALUS

\begin{tabular}{|l|c|c|c|}
\hline & $\begin{array}{c}\text { Sensitivity } \\
(\boldsymbol{\%})\end{array}$ & $\begin{array}{c}\text { Specificity } \\
(\boldsymbol{\%})\end{array}$ & $\begin{array}{c}\text { Accuracy } \\
(\boldsymbol{\%})\end{array}$ \\
\hline Baseline & 60.61 & 58.33 & 59.42 \\
\hline Infusion & 84.85 & 36.11 & 59.42 \\
\hline Plateau & 63.64 & 50.00 & 56.52 \\
\hline Recovery & 39.39 & 75.00 & 57.97 \\
\hline
\end{tabular}

Sensitivity: \% of patients with normal pressure hydrocephalus correctly classified.

Specificity: $\%$ of patients having developed a secondary form normal pressure hydrocephalus correctly classified.

Accuracy: \% of total patients correctly classified.

[3] M. Czosnyka, Z. Czosnyka, S. Momjian, and J. D. Pickard, "Cerebrospinal fluid dynamics," Physiol. Meas., vol. 25, no. 5, pp. R51-R76, 2004.

[4] R. Katzman and F. Hussey "A simple constant-infusion manometric test for measurement of CSF absorption," Neurology, vol. 20, no. 6, pp. 534-544, 1970.

[5] M. Czosnyka and J. D. Pickard, "Monitoring and Interpretation of Intracranial Pressure," J. Neurol. Neurosur. Ps., vol. 75, no. 6, pp. 813-821, 2004.

[6] D. Santamarta, R. Hornero, D. Abásolo, M. Martínez-Madrigal, J. Fernández, and J. García-Cosamalón, "Complexity analysis of the cerebrospinal fluid pulse waveform during infusion studies," Childs Nerv. Syst., vol. 26, no. 12, pp. 1683-1689, 2010.

[7] C. Bandt and B. Pompe, "Permutation Entropy: A Natural Complexity Measure for Time Series," Phys. Rev. Lett., vol. 88, no. 17, pp. (174102-1)-(174102-4), 2002.

[8] Z. H. Czosnyka, K. Cieslicki, M.Czosnyka, and J. D. Pickard, "Hydrocephalus shunts and waves of intracranial pressure," Med. Biol. Eng. Comput., vol. 43, no. 1, pp. 71-77, 2005.

[9] C. Bian, C. Qin, Q. D. Y. Ma, and Q. Shen, "Modified permutationentropy analysis of heartbeat dynamics," Phys. Rev. E, vol. 85, no. 2, pp. (021906-1)-(021906-7), 2012.

[10] Y. Cao, W.-W. Tung, J. B. Gao, V. A. Protopopescu, and L.M. Hively, "Detecting dynamical changes in time series using permutation entropy," Phys. Rev. E, vol. 70, no. 4, pp. (046217-1)(046217-7), 2004.

[11] J. Li and J. P. Fine, "ROC analysis with multiple classes and multiple tests: methodology and its application in microarray studies," Biostatistics, vol. 9, no. 3, pp. 566-576, 2008.

[12] K. H. Zou, J. O'Malley, and L. Mauri, "Receiver-Operating Characteristic Analysis for Evaluating Diagnostic Tests and Predictive Models," Circulation, vol. 115, no. 5, pp. 654-657, 2007.

[13] M. Soehle, B. Gies, P. Smielewski, and M. Czosnyka, "Reduced complexity of intracranial pressure observed in short time series of intracranial hypertension following traumatic brain injury in adults," J. Clin. Monitor. Comp., vol. 27, no. 4, pp. 395-403, 2013.

[14] R. Hornero, M. Aboy, D. Abásolo, J. McNames, W. Wakeland, and B. Goldstein, 2006 "Complex analysis of intracranial hypertension using approximate entropy”, Crit. Care Med., vol. 34, no. 1, pp. 8795, 2006.

[15] M. Zanin, L. Zunino, O.A. Rosso, and D. Papo, "Permutation entropy and its main biomedical and econophysics applications: A review," Entropy, vol. 14, pp. 1553-1577, 2012.

[16] B. Fadlallah, B. Chen, A. Keil, and J. Principe, 'Weighted-permutated entropy: A complexity measure for time series incorporating amplitude information," Phys. Rev. E, vol. 87, no. 2, pp. (022911-1)(022911-7), 2013.

[17] A. Porta, V. Bari, A. Marchi, B. De Maria, P. Castiglioni, M. di Rienzo, S. Guzetti, A. Cividjian, and L. Quintin, "Limits of permutation-based entropies in assessing complexity of short heart period variability," Physiol. Meas., vol. 36, no. 4, pp. 755-765, 2015. 